\title{
Integral analyses of survival-related long non-coding RNA MIR210HG and its prognostic role in colon cancer
}

\author{
ZHAOHUI RUAN $^{1 *}$, ZIXUAN XU $^{1 *}$, ZHAOZHI LI $^{2}$ and YANNI LV ${ }^{1}$ \\ ${ }^{1}$ Pharmacy Department, The First Affiliated Hospital of Nanchang University, Nanchang, Jiangxi 330006; \\ ${ }^{2}$ Key Laboratory of Bio-resources and Eco-environment, College of Life Sciences, \\ Sichuan University, Chengdu, Sichuan 610000, P.R. China
}

Received May 1, 2018; Accepted April 15, 2019

DOI: $10.3892 / 01.2019 .10435$

\begin{abstract}
Colon cancer is the third most common cancer worldwide, and lymphatic metastasis is one of the principal factors affecting patient prognosis. Recent studies have revealed that long non-coding RNAs (lncRNAs) serve as important regulators in the pathogenesis of colon cancer, therefore affecting patient survival rates. In the present study, colon cancer-associated lncRNAs were screened based on their influence on patient survival. A number of survival-associated lncRNAs (and their potential mechanisms of action) were identified, with the strongest candidate being MIR210HG. Gene expression correlation and protein-protein interaction (PPI) network analyses were performed to identify MIR210HG-associated genes. Various bioinformatics analyses (including gene ontology and Kyoto Encyclopedia of Genes and Genomes analyses) were conducted to investigate the prognostic role of MIR210HG and its associated genes, in colon cancer. Higher expression levels of MIR210HG were associated with shorter overall survival in patients with colon cancer, which was significant in 373 candidates. Multiple findings indicated that MIR210HG may exert its effects in colon cancer through the modulation of energy metabolism and cell
\end{abstract}

Correspondence to: Dr Yanni Lv, Pharmacy Department, The First Affiliated Hospital of Nanchang University, 17 Yongwaizheng Street, Nanchang, Jiangxi 330006, P.R. China

E-mail: lvyanni@126.com

*Contributed equally

Abbreviations: lncRNA, long non-coding RNA; DHFR, dihydrofolate reductase; TCGA, The Cancer Genome Atlas; ALDOA, fructose-bisphosphate aldolase A; PKM, pyruvate kinase M; DAVID, Database for Annotation, Visualization, and Integrated Discovery; GO, gene ontology; KEGG, Kyoto Encyclopedia of Genes and Genomes; PPI, protein-protein interaction; STRING, Search Tool for the Retrieval of Interacting Genes/Proteins; ClusterONE, Clustering with Overlapping Neighborhood Expansion

Key words: colon cancer, IncRNA, MIR210HG, The Cancer Genome Atlas, energy metabolism, cell adhesion adhesion. Further predictions suggested that MIR210HG may affect colon cancer via transcription and post-transcriptional processing. Collectively, these results provided evidence of a transcriptional regulatory network of MIR210HG in colon cancer, and suggested its potential role as a novel biomarker and therapeutic target for colon cancer.

\section{Introduction}

Colon cancer is the third most common type of cancer, and the leading cause of cancer-related mortality worldwide (1). There is increasing evidence to suggest that the progression of colon cancer is a multiple-step process, involving genetic and epigenetic abnormalities (2). By comparison, epigenetic alterations are more frequently observed, and carry greater effects compared with gene mutations. For example, the silencing micro RNA-137 can target and decrease the expression level of $>500$ protein coding genes (3).

Long non-coding RNAs (lncRNAs) are a type of non-coding transcript of $>200$ nucleotides in length. IncRNAs have been associated with a number of physiological and pathological processes in various human diseases, regulating gene expression via epigenetic, transcriptional and post-transcriptional modification (4). Recent studies revealed that IncRNA was an important regulator in colon cancer pathogenesis, modulating numerous processes including cell proliferation, differentiation, tumor migration, invasion and angiogenesis. It has been reported that IncRNA SUMO1P3 stimulated the proliferation, metastasis and angiogenesis of colon cancer cells, suggesting it's potential as a novel prognostic indicator and therapeutic target for the disease (5).

lncRNAs can target different steps in the transcription process, regulating the activation or regression of gene transcription by modulating different transcriptional components, including RNA polymerase (RNAP) II and the DNA duplex (6). These lncRNAs may be part of the regulatory network that, together with transcription factors, regulate the level of gene expression. Reportedly, certain lncRNAs acted as regulators of general transcription factors which were required for the activity of RNAP II (7). These transcription factors, in addition to components of the initiation complex, assemble at promoter regions and participate in transcription elongation and the regulation of cis elements (8); it was reported that an 
lncRNA transcribed from the dihydrofolate reductase (DHFR) gene was able to form a RNA-DNA triplex in the promoter region of DHFR, inhibiting its transcription by blocking the binding of transcription initiation factor IIB (9). Furthermore, the gene coding lncRNA ANRIL is able to run antisense, silencing P15 with which it overlaps.

However, survival-related lncRNAs, particularly in colon cancer remained to be identified. In order to select therapeutically relevant lncRNAs, a linear regression model was used to identify survival-related lncRNAs in colon cancer and further detect MIR210HG-related genes. Gene regulatory network and pathway analyses revealed that MIR $210 \mathrm{HG}$ may exert its effects in colon cancer through the regulation of cancer cell metabolism and adhesion. Furthermore, bioinformatics predictions indicated that MIR210HG may affect colon cancer by regulating transcription and post-transcriptional processing.

\section{Materials and methods}

Screening of survival-related IncRNAs in colon cancer. The RNA-seq data of colon cancer-associated lncRNAs, which consisted of 471 tumor samples and 41 normal samples, were downloaded from The Cancer Genome Atlas (TCGA) data portal (https://cancergenome.nih.gov/) (10). The data were divided into high and low expression groups according to a cut-off of 75 and $25 \%$, respectively. Homo_sapiens. GRCh38.84 was used for annotation. The RNA-seq data of other gastrointestinal cancers, including esophageal cancer (162 tumor and 11 normal samples), rectal cancer (167 tumor and 10 normal samples) and stomach cancer (375 tumor and 32 normal samples), were also downloaded, and assigned into high and low expression groups using the same classification criteria as in colon cancer. The association between lncRNA expression and patient survival was determined using the Kaplan-Meier method, and evaluated using the log-rank test. The R package survival (https://github.com/therneau/survival) was used to perform these statistical analyses. $\mathrm{P}<0.05$ was set as the significance threshold.

Genes significantly associated with MIR210HG expression in colon cancer. Based on the above screening, the most significant survival-related lncRNA in colon cancer was identified as MIR210HG; this lncRNA was therefore selected for use in further analysis. Associations between the expression of MIR210HG and other genes were calculated using a linear regression model. It was hypothesized that genes whose expression levels were linearly associated with MIR201HG were likely to be located nearby, upstream or downstream. This method was widely used to screen target-related genes. RNA expression was processed using $\log _{2}($ counts +1$)$ prior to analysis. Subsequently, multiple selection criteria (P-value and adj. $\mathrm{r}^{2}$ ) were applied to determine the most appropriate candidates. $\mathrm{P}<0.05$ and adj. $\mathrm{r}^{2}>0.16$ were set as the significance threshold. $\mathrm{R}$ was used to perform these statistical analyses.

Functional and pathway enrichment analysis. Gene ontology (GO) enrichment analysis (http://www.geneontology.org/) was used to detect biological processes, molecular functions and cellular components. Kyoto Encyclopedia of Genes and
Genomes (KEGG) analysis (http://www.kegg.jp/), which is used for pathway investigations, was performed using the Database for Annotation, Visualization, and Integrated Discovery (DAVID; https://david.ncifcrf.gov/, version 6.8) (11-13). $\mathrm{P}<0.05$ was set as the significance threshold.

Investigation of the correlation between MIRE210HG expression and lymphatic metastasis. All clinical data were obtained from TCGA. A normality test, the Kolmogorov-Smirnov test, was performed and a Mann Whitney $U$ test was subsequently used to assess the association between MIRE210HG expression and metastasis in colon cancer. $\mathrm{P}<0.05$ was considered to indicate a statistically significant difference. SPSS Statistics 22.0 (IBM Corp.) was used to perform statistical analysis.

Protein-protein interaction (PPI) network construction and identification of hub genes. All MIR210HG-related genes were imported into the Search Tool for the Retrieval of Interacting Genes/Proteins (STRING) to construct a PPI network (14). A combined score $\geq 0.4$ was set as the significance threshold. The network was visualized using Cytoscape (version 3.5.1, http://www.cytoscape.org/) (15). The top 10 hub genes were filtered using cytoHubba, an app in Cytoscape, using the Maximal Clique Centrality (MCC) method (16).

Module analysis of the PPI network. To investigate the most significant modules, Clustering with Overlapping Neighborhood Expansion (ClusterONE), an app in Cytoscape, was used to screen modules within the PPI network with a minimum size of 6 , and the minimum density set to auto (17). $\mathrm{P}<0.05$ was considered to indicate a statistically significant difference. GO and KEGG analyses of genes in the screened modules were performed using DAVID.

Transcription and post-transcriptional modulation prediction. To further investigate the mechanism of MIR210HG in colon cancer, known MIR210HG-protein interactions were summarized using starBase v2.0 (http://starbase.sysu. edu.cn/index.php) (18). Novel lncRNA-protein interactions between MIR210HG and its associated transcription factors were also predicted using RPIseq (http://pridb.gdcb.iastate. edu/RPISeq/index.html) (19). Candidates with a Random Forest classifier value $>0.5$ and Support Vector Machine classifier value $>0.5$ were considered to be positive results.

Interaction probabilities generated using RPISeq range between 0 and 1 . In performance evaluation experiments, predictions with probabilities $>0.5$ were considered 'positive', indicating that the corresponding RNA and protein were likely to interact. Neighboring mRNAs located within $1 \mathrm{Mbps}$ of the MIR210HG loci were investigated to predict possible cis target genes of MIR210HG. Candidate gene information was downloaded from USCS (GRCh38) (20).

\section{Results}

MIR210HG expression correlates with survival in patients with colon cancer. IncRNAs have been reported to contribute to patient survival in multiple cancers, including colon cancer (20), breast cancer (21) and larynx squamous cell carcinoma (22), and therefore, in the present study, survival-related 
Table I. Top 10 survival-related lncRNAs in colon cancer.

\begin{tabular}{lcc}
\hline & $\begin{array}{c}\text { Expression level } \\
{\left[\log _{2}(\text { counts }+1)\right]}\end{array}$ & P-value \\
\hline RP11-66B24.2 & $4.35(1.58,8.63)$ & $1.08 \times 10^{-4}$ \\
MIR210HG & $208.82(111.73,352.98)$ & $1.19 \times 10^{-4}$ \\
RP11-367H1.1 & $5.29(2.42,9.54)$ & $1.20 \times 10^{-4}$ \\
CTC-573N18.1 & $0.68(0.08,2.9)$ & $1.30 \times 10^{-4}$ \\
LA16c-380A1.1 & $1.36(0.14,3.41)$ & $1.31 \times 10^{-4}$ \\
RP11-108K3.1 & $7.79(4.3,13.05)$ & $1.35 \times 10^{-4}$ \\
RP11-93I21.3 & $0.52(0.08,2.15)$ & $1.49 \times 10^{-4}$ \\
LINC00174 & $179.71(122.2,269.39)$ & $2.11 \times 10^{-4}$ \\
RP11-108K3.2 & $5.38(1.97,9.91)$ & $2.96 \times 10^{-4}$ \\
AP006621.5 & $68.84(44.66,95.88)$ & $3.80 \times 10^{-4}$ \\
\hline
\end{tabular}

lncRNA, long no-coding RNA.

IncRNAs in colon cancer were investigated. RNA-seq and clinical data from colon cancer cohorts (obtained from TCGA) were analyzed to assess the association between various lncRNAs and patient survival. A total of 226 survival-related lncRNAs were selected using $\mathrm{P}<0.05$ as the cut-off threshold (The top 10 survival-related lncRNAs are presented in Table I). A number of lncRNAs were reported to contribute to tumor promotion, including MIR210HG $\left(\mathrm{P}=1.19 \times 10^{-3}\right.$; log-rank test), which is a diagnostic biomarker in glioma (23). A high expression level of MIR210HG was significantly associated with shorter overall survival in patients with colon cancer, suggesting a potential prognostic role for MIR210HG in the disease (Fig. 1A). However, MIR210HG showed a weak association with survival from esophageal, rectal and stomach cancers, indicating that this particular effect of MIR210HG overexpression is specific to colon cancer (Fig. 1).

Investigation of MIR210HG-related genes in colon cancer. TCGA RNA-seq expression data for MIR210HG was downloaded, and the correlation between MIR210HG expression and other genes in colon cancer was calculated using a linear regression model. A total of 373 genes with $\mathrm{P}<0.05$ and adj. $\mathrm{r}^{2}>0.16$ were filtered out for further analysis (Fig. 2). The top 10 genes significantly associated with MIR210HG expression in colon cancer are presented in Table II. A total of 231 and 142 genes were positively, and negatively associated with MIR210HG expression in colon cancer, respectively. Additionally, the known PPIs among these MIR210HG-related candidates were detected using STRING (Fig. 3). A total of 359 nodes were noted in the network, and their interactions are presented in Fig. 3A. To identify the key nodes that most affected this network, MCC analyses were conducted to rank the strongest hub genes. Hub genes act as cores within the regulatory network, and the results revealed complex and strong links with various MIR210HG-related candidates. It was speculated that the expression of these hub genes may be directly regulated by MIR $210 \mathrm{HG}$ or its downstream targets, and the results of linear regression analysis showed a significant correlation between the expression of the $10 \mathrm{hub}$ genes and MIR210HG (Table III). Modules were regarded
Table II. Top 10 genes significantly associated with MIR210HG expression in colon cancer.

\begin{tabular}{lcc}
\hline Gene & P-value & Adj.r ${ }^{2}$ \\
\hline NDRG1 & $7.96 \times 10^{-51}$ & 0.38 \\
ALDOA & $1.43 \times 10^{-51}$ & 0.38 \\
EGLN3 & $2.49 \times 10^{-51}$ & 0.36 \\
ALDOC & $1.09 \times 10^{-51}$ & 0.35 \\
SLC16A3 & $5.78 \times 10^{-51}$ & 0.34 \\
PFKFB4 & $3.23 \times 10^{-51}$ & 0.33 \\
MIR210 & $1.02 \times 10^{-51}$ & 0.30 \\
SLC6A8 & $1.85 \times 10^{-51}$ & 0.30 \\
NARF & $3.34 \times 10^{-51}$ & 0.30 \\
ANGPTL4 & $7.93 \times 10^{-51}$ & 0.30 \\
\hline
\end{tabular}

NDRG1, N-myc downstream regulated 1; ALDOA, aldolase, fructose-bisphosphate A; EGLN3, egl-9 family hypoxia inducible factor 3; ALDOC, aldolase, fructose-bisphosphate C; PFKFB4, 6-phosphofructo-2-kinase/fructose-2,6-biphosphatase 4; MIR210, microRNA 210; SLC6A8, solute carrier family 6 member 8; NARF, nuclear prelamin A recognition factor; ANGPTL4, angiopoietin like 4.

as a group of proteins that participate in the same biological process, or composition of the same complex (24). Modularity analysis was conducted, and 2 modules were selected from the constructed PPI network, as illustrated in Fig. 3B and C. GO and KEGG analysis indicated that module 1 may be associated with glycolysis and cell adhesion (e.g. triosephosphate isomerase 1, GAPDH, enolase 2, mucin 1, cell surface associated, egl-9 family hypoxia inducible factor 3, Bardet-Biedl syndrome 10 , and phosphofructokinase, liver type), while module 2 may be associated with ubiquitination (e.g. kelch like family member 42 , anaphase promoting complex subunit 2 , F-box and WD repeat domain containing 5, and F-box and leucine rich repeat protein 15) (Figs. 4B and C; 5B).

MIR210HG may impact colon cancer by targeting energy metabolism and cell adhesion. In order to determine the possible mechanism of MIR210HG in colon cancer, GO and KEGG pathway analyses were conducted. The GO results revealed that MIR210HG expression was strongly associated with energy metabolism in colon cancer, as evidenced by the enrichment of genes involved in canonical glycolysis $\left(\mathrm{P}=1.15 \times 10^{-8}\right)$, glycolytic process $\left(\mathrm{P}=2.78 \times 10^{-5}\right)$ and gluconeogenesis $(\mathrm{P}=0.001)$ (Fig. 4A). Additionally, the KEGG results revealed that MIR $210 \mathrm{HG}$ was significantly associated with the biosynthesis of glycolysis or gluconeogenesis $\left(\mathrm{P}=8.33 \times 10^{-6}\right)$ and central carbon metabolism in cancer $(\mathrm{P}=0.003)$ (Fig. 5A). Furthermore, cell-cell adhesion $\left(\mathrm{P}=3.99 \times 10^{-4}\right)$, cell-cell adherens junction $(\mathrm{P}=0.002)$ and cadherin binding involved in cell-cell adhesion $(\mathrm{P}=0.001)$ were notably enriched in biological process, cellular component and molecular function analysis, respectively. A recently published paper suggested that MIR210HG facilitates cancer cell invasion and metastasis in osteosarcoma (25), which is consistent with the findings of the present study. These findings suggest that MIR210HG may exert its effects in colon 

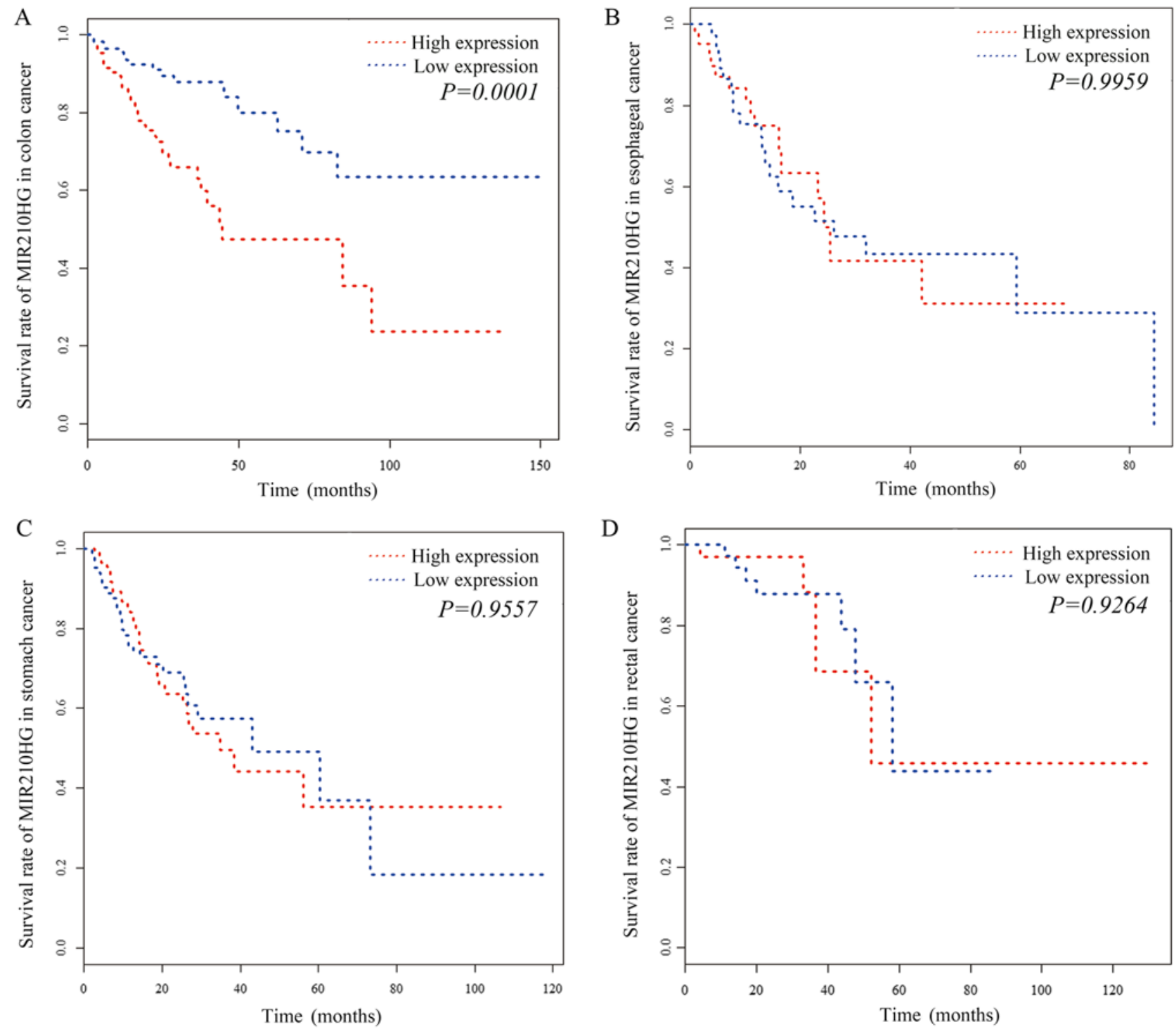

Figure 1. MIR210HG survival curves for gastrointestinal cancers. (A) MIR210HG survival curve for colon cancer ( $\mathrm{P}=1.19 \mathrm{x} 10^{-3}$; log-rank test). (B) MIR210HG survival curve for esophageal cancer ( $\mathrm{P}=1.00$; log-rank test). (C) MIR210HG survival curve for stomach cancer ( $\mathrm{P}=0.96$; log-rank test). (D) MIR210HG survival curve for rectal cancer $(\mathrm{P}=0.93$; log-rank test).

cancer by modulating energy metabolism and cell adhesion, which are closely associated with the prognosis of patients with colon cancer. Furthermore, a Mann Whitney U test was performed to verify the association between lymphatic metastasis and MIR210HG expression in colon cancer, and a strong association was identified $(\mathrm{P}=0.02$; Fig. 6). These results suggest that MIR210HG may be a modulator of colon cancer cell metastasis, and are consistent with the results of the GO and KEGG enrichment analyses.

MIR210HG may influence colon cancer via transcription and post-transcriptional processing. To further investigate the mechanism of MIR210HG in colon cancer, various possible interactions between MIR210HG and screened MIR210HG candidates were predicted. Known MIR210HG-protien interactions were summarized using starBase v2.0. Specific genes associated with RNA splicing and degradation were detected (Table IV), including FUS RNA binding protein (FUS). These genes may therefore serve as post-transcriptional regulators in colon cancer. Novel lncRNA-protein interactions were also predicted using RPISeq. Since transcription factors are considered to be important regulators of gene expression, the focus was on their association with MIR210HG (Table V). Predictions for Mediator of RNA polymerase II transcription (MED)15, MED16 and B-cell lymphoma 3 protein (BCL3; probabilities $>0.5$ ) were considered to be 'positive'. It was observed that MED16 and BCL3 were connected to hub genes within the MIR210HG regulatory network, suggesting that they may modulate the network by stimulating these genes.

lncRNAs also interacted with other cis acting elements of promoters or co-expressed genes, thus regulating gene expression via transcription or post-transcriptional processes. We therefore investigated neighboring mRNAs located within $1 \mathrm{Mbps}$ of the MIR210HG locus to predict possible cis target genes. A total of 13 genes were identified (Table VI), 5 of which were members of the MIR210HG regulatory network. One of these genes was HRAS, which is known to be involved 
Table III. Top 10 hub genes in protein-protein interaction networks.

\begin{tabular}{rlcc}
\hline No. & Hub genes & P-value & Adj.r.squared \\
\hline 1 & PKM & $6.14 \times 10^{-26}$ & 0.21 \\
2 & TPI1 & $1.01 \times 10^{-20}$ & 0.17 \\
3 & PFKL & $1.18 \times 10^{-25}$ & 0.21 \\
4 & PFKP & $9.47 \times 10^{-27}$ & 0.22 \\
5 & GAPDH & $1.65 \times 10^{-21}$ & 0.17 \\
6 & ENO2 & $7.55 \times 10^{-27}$ & 0.22 \\
7 & LDHA & $1.88 \times 10^{-29}$ & 0.24 \\
8 & ALDOA & $1.43 \times 10^{-50}$ & 0.38 \\
9 & ALDOC & $1.09 \times 10^{-46}$ & 0.35 \\
10 & PFKFB4 & $3.23 \times 10^{-42}$ & 0.33 \\
\hline
\end{tabular}

PKM, pyruvate kinase M1/2; TPI1, triosephosphate isomerase 1; PFKL, phosphofructokinase, liver type; PFKP, phosphofructokinase, platelet; GAPDH, glyceraldehyde-3-phosphate dehydrogenase; ENO2, enolase 2; LDHA, lactate dehydrogenase A; ALDOA, aldolase, fructose-bisphosphate A; ALDOC, aldolase, fructose-bisphosphate C; PFKFB4, 6-phosphofructo-2-kinase/fructose-2,6-biphosphatase 4.

Table IV. Known MIG210HG-protien interactions via starBase v2.0.

\begin{tabular}{lcccr}
\hline Name & IncRNA & $\begin{array}{c}\text { Target } \\
\text { sites }\end{array}$ & BioComplex & ClipReadNum \\
\hline eIF4AIII & MIR210HG & 12 & 2 & 31 \\
FUS & MIR210HG & 1 & 1 & 5 \\
SFRS & MIR210HG & 1 & 1 & 800 \\
U2AF65 & MIR210HG & 1 & 1 & 2 \\
\hline
\end{tabular}

BioComplex is the number of supporting experiments. ClipReadNum is the read number of CLIP-Seq. IncRNA, long non-coding RNA; eIF4AIII, Eukaryotic initiation factor 4A-III; FUS, FUS RNA binding protein; SFRS, Serine/arginine-rich splicing factor 1; U2AF65, U2 small nuclear RNA auxiliary factor 2 .

in central carbon metabolism in cancer. HRAS is a member of the Ras superfamily of GTPases, which are involved in the regulation of cell division in response to growth factor stimulation. Activated mutant HRAS (Hras-V12) is able to initiate irreversible cell cycle arrest and increase the expression of the tumor suppressors p16 and p53 (26).

\section{Discussion}

Lymphatic metastasis is one of the principal factors to affect the prognosis of patients with colon cancer (27). The survival rates of patients with lymphatic metastasis is significantly lower compared with that of patients without lymphatic metastasis (28). Furthermore, it has been identified that metastasis-related factors may be used as prognostic biomarkers or potential therapeutic targets for improving the survival rates of patients with colon cancer (29-31).
In the present study, candidate lncRNAs were screened based on the effects of lncRNA expression on the survival rate of patients with colon cancer. The employed screening method may be more suitable for clinical practice compared with traditional selection methods, and is more likely to be applied to treatment. The same method was used to screen survival-related lncRNAs in breast cancer, and a co-expressed gene network of the IncRNA with the greatest effect, LINC00704 (data not shown), was also constructed. The results of GO and KEGG analyses suggested that LINC00704-related genes are associated with the regulation of cell proliferation, which is in agreement with a previous report (32).

Glycolysis is the primary method by which tumor cells generate energy, even under normoxic conditions (33). Glycolysis provides energy for tumor cells, and so targeting glycolysis may have potential as a method to control tumor proliferation. Changes in glycolysis in tumor cells are associated with resistance to anticancer drugs. For instance, Song et al (34) reported that increased glycolytic activity and low phosphorylation efficiency increases chemo-resistance in patients with acute myeloid leukemia. This may be regulated by glycolysis-related molecules, including hypoxia-inducible factor $1 \alpha$, hexokinase II, glucose transport 1 and lactate dehydrogenase (34).

In the present study, a number of survival-related lncRNAs in colon cancer were identified. Notably, the upregulation of a tumor promoter IncRNA, MIR210HG, was associated with shorter overall patient survival. To the best of our knowledge, current investigations into the association between MIR210HG and colon cancer prognosis are limited. Here, linear regression analysis was performed and an MIR210HG-related regulatory network was constructed. Specific MIR210HG-related candidates have been reported to be associated with colon cancer cell survival and metastasis, suggesting a possible link between MIR210HG expression and these genes that affects overall survival in patients with colon cancer. MIR210HG also influences osteosarcoma metastasis (25); furthermore, it was revealed that patients with high MIR210HG expression levels were more likely to have lymphatic metastasis. Genes that were not reported may be regarded as novel colon cancer-specific hubs in the MIR210HG regulatory network. Two notable modules and the top 10 hub genes were screened out using modularity analysis and MCC. A number of these hub genes, including fructose-bisphosphate aldolase A (ALDOA) and pyruvate kinase $\mathrm{M}(\mathrm{PKM}) 2$, were associated with glycolysis, cell migration and apoptosis in colon cancer cells. These hub genes have been demonstrated to affect colon cancer prognosis. For instance, ALDOA was reported to be a key promoting element in colon cancer; ALDOA acts as a significant regulator of hypoxic adaptation in colon cancer cells (35), whilst it has also been reported to contribute to metastasis of colon cancer. Another key hub gene, PKM2 was able to disturb the Warburg effect and increase the sensitivity of cancer cells to tumor necrosis factor-related apoptosis-inducing ligand-induced cell death. Yang et al (36) reported that PKM2 overexpression was able to modulate STAT3 signaling, thereby facilitating the migration of colorectal cancer cells (36). Of these genes, ALDOA was found to have the strongest correlation with MIR210HG (adj. $\mathrm{r}^{2}=0.4296 ; \mathrm{P}=7.450 \times 10^{-55}$ ), which suggests that it may be a direct target of MIR210HG. 
Table V. Prediction of lncRNA-protein interactions between MIR210HG and candidate transcription factors through RPIseq.

\begin{tabular}{lclcc}
\hline Transcription factors & Isoform & \multicolumn{1}{c}{ Gene names } & RF classifier & SVM classifier \\
\hline MED15 & 1 & Mediator complex subunit 15 & 0.9 & 0.57 \\
MED15 & 2 & Mediator complex subunit 15 & 0.9 & 0.9 \\
MED15 & 3 & Mediator complex subunit 15 & 0.8 & 0.59 \\
MED16 & 1 & Mediator complex subunit 16 & 0.75 & 0.61 \\
MED16 & 2 & Mediator complex subunit 16 & 0.75 & 0.61 \\
MED16 & 3 & Mediator complex subunit 16 & 0.8 & 0.6 \\
MED16 & 4 & Mediator complex subunit 16 & 0.8 & 0.85 \\
MED16 & 5 & Mediator complex subunit 16 & 0.85 & 0.48 \\
CDK9 & 1 & Cyclin dependent kinase 9 & 0.75 \\
CDK9 & 2 & Cyclin dependent kinase 9 & 0.55 \\
BCL3 & 1 & B-cell CLL/lymphoma 3 & 0.51 \\
PPP1R13L & 1 & Protein phosphatase 1 regulatory subunit 13 like & 0.53 \\
\hline
\end{tabular}

RF, Random forrest; SVM, Support Vector Machine.

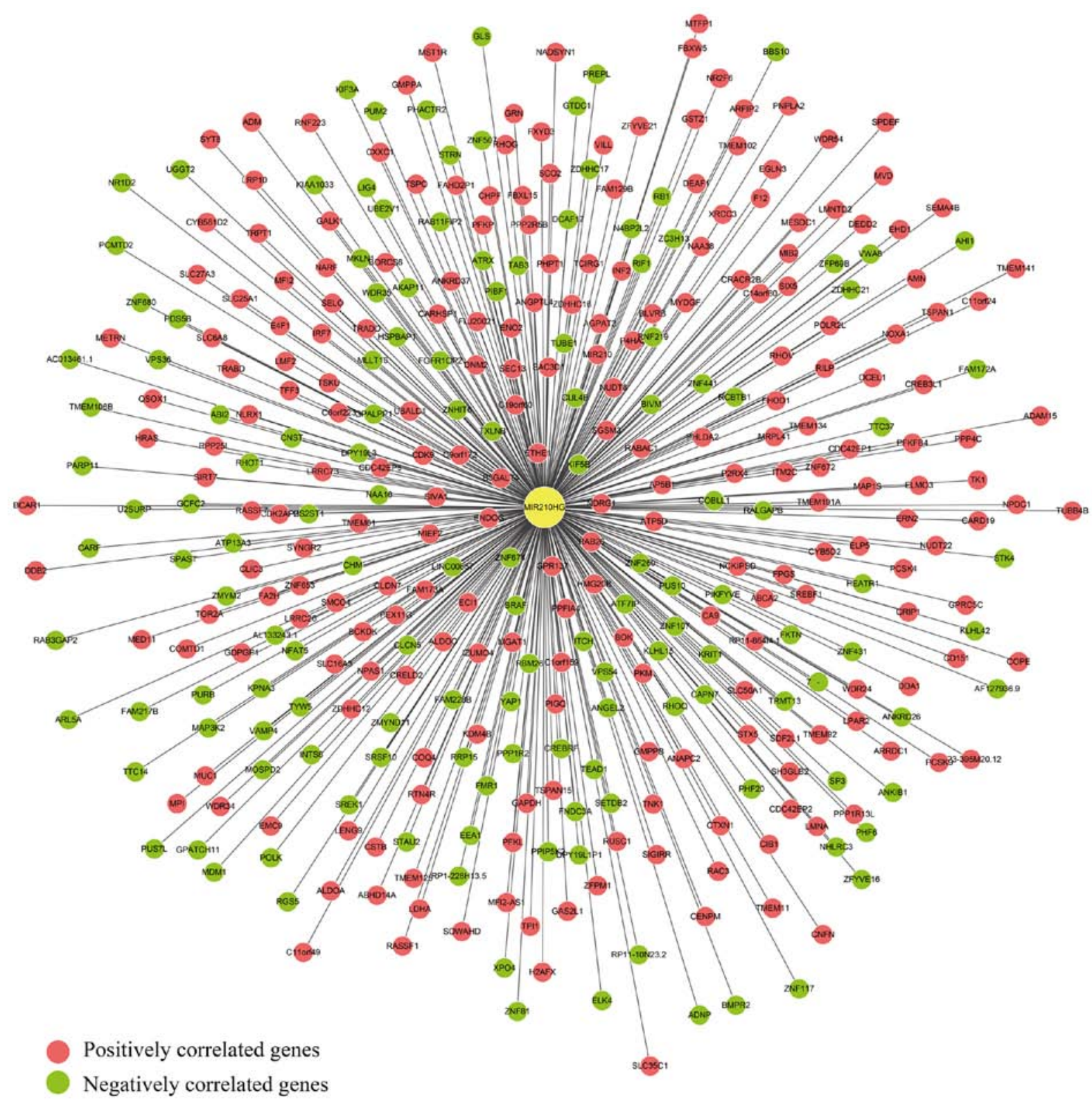

Figure 2. Genes significantly associated with MIR210HG expression in colon cancer. 
A

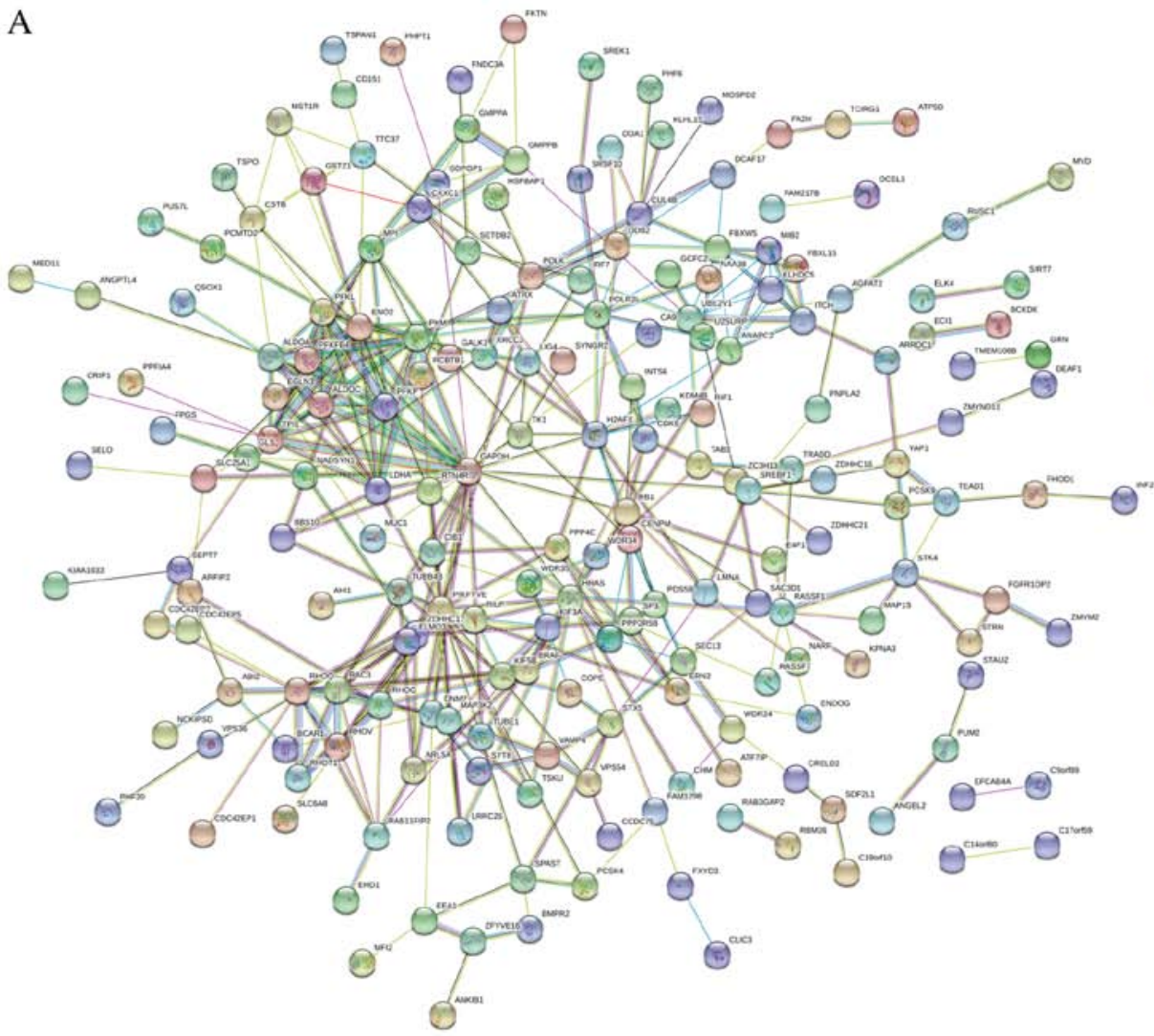

B

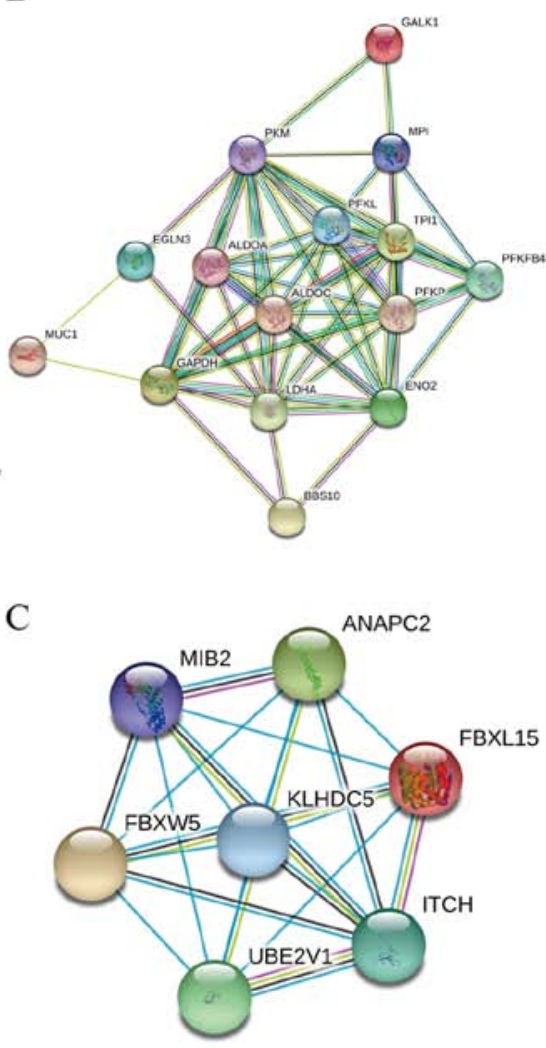

Figure 3. PPI network and modules. (A) Known PPIs among MIR210HG-related candidates. (B) Module 1 and (C) module 2. PPI, protein-protein interaction.

A

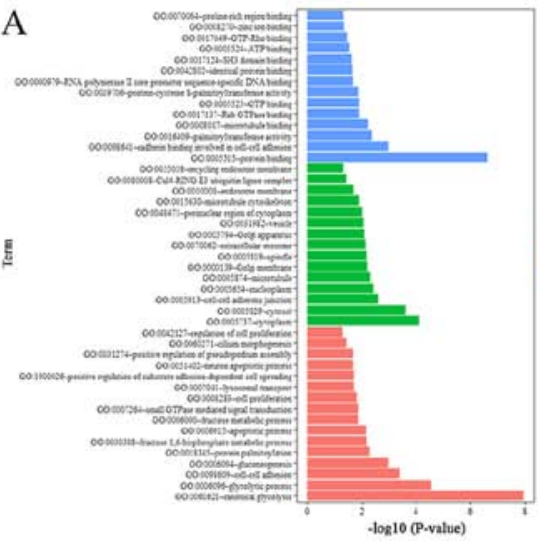

B

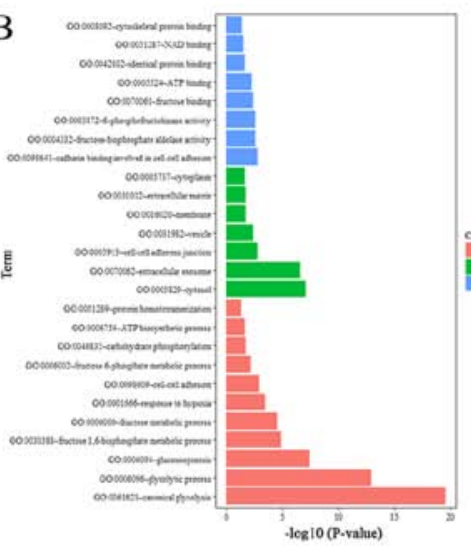

C

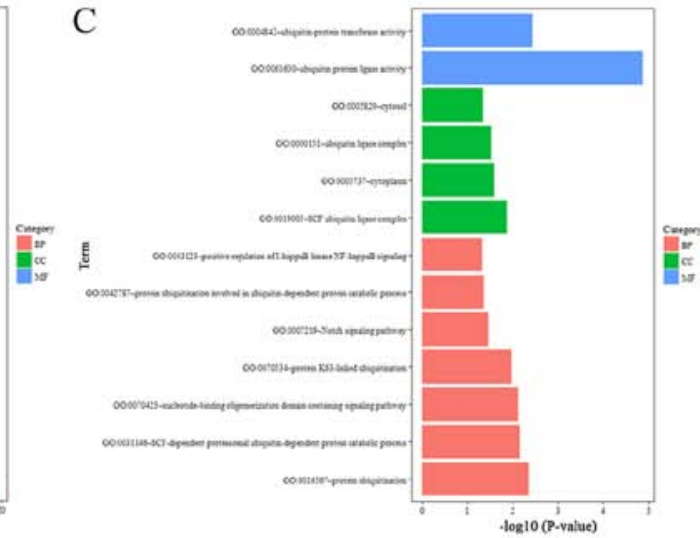

Figure 4. GO functional enrichment analyses. (A) GO functional enrichment analyses of MIR210HG-related candidates. GO functional enrichment analyses results for (B) module1 and (C) module 2. GO, gene ontology.

The possible mechanism of interaction between MIR210HG and its co-expressed genes was also predicted. Reports have indicated that MIR210HG may affect colon cancer via transcription and post-transcriptional processing. Certain genes associated with RNA splicing and degradation, including FUS, were reported to interact with MIR210HG, suggesting that they may serve as post-transcriptional regulators in colon cancer. mRNA splicing is a vital step in post-transcriptional regulation, as it can trigger translation and functionally diversify proteins. In part of a study by Zarnack et al (37) experimental verification was carried out and reported a correlation between MIR210HG and U2AF65 (supplementary material; summary by starBase v2.0 database: http://starbase.sysu.edu.cn/index. php). While, according to the present study, MIR210HG and U2AF65 also showed associations (probabilities $>0.5$ using RPISeq: http://pridb.gdcb.iastate.edu/RPISeq/index.html), which is consistent with Zarnack's experimental results. However, in order to develop novel treatment methods to improve the prognosis of patients with colon cancer, we must improve our understanding of the molecular mechanisms of IncRNAs. In the present study, novel lncRNA-protein interactions were detected using RPISeq, and numerous transcription factors (including MED15, MED16 and BCL3) were considered to be positive candidates. In particular, MED16 and BCL3 

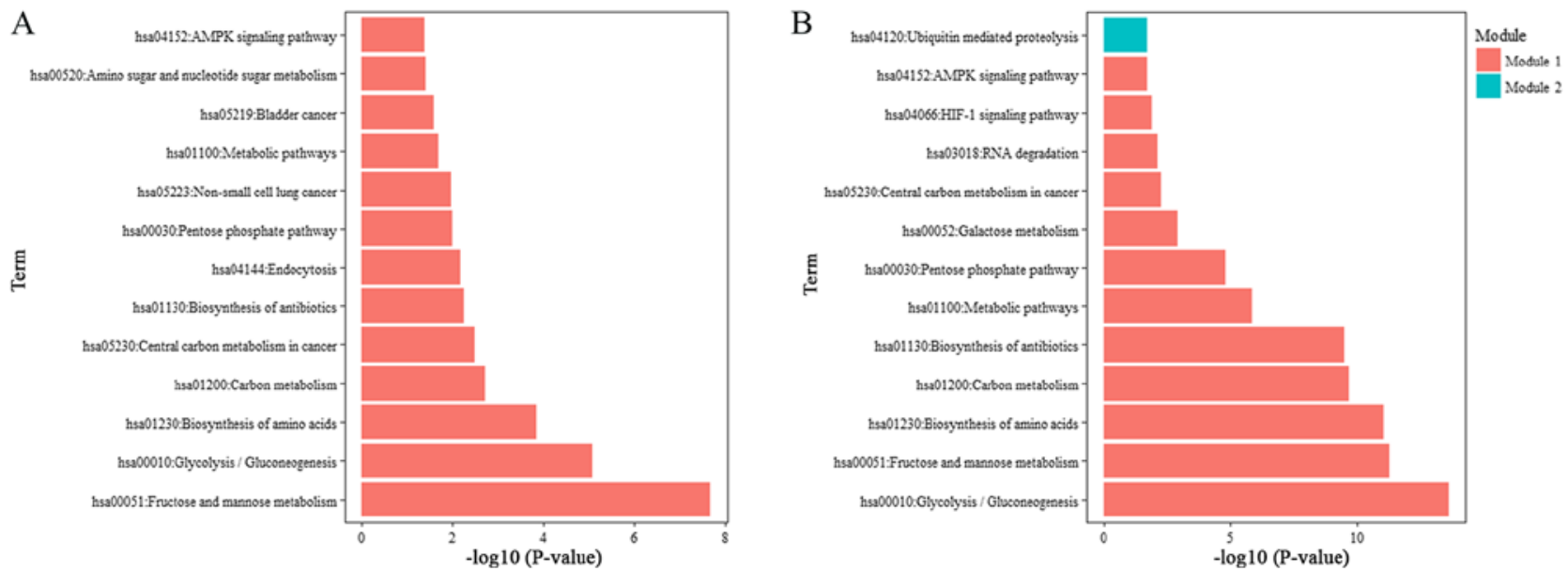

Figure 5. KEGG pathway enrichment analyses. (A) KEGG pathway enrichment analyses of MIR210HG-related candidates. (B) KEGG pathway enrichment analyses results of modules. KEGG, Kyoto Encyclopedia of Genes and Genomes.

Table VI. Prediction of nearby cis target genes of MIR210HG.

Nearby gene names

Whether in PPI network

\begin{tabular}{ll}
\hline RNH1 & 0 \\
LOC101059906 & 0 \\
HRAS & 1 \\
LRRC56 & 0 \\
LMNTD2 & 1 \\
RASSF7 & 1 \\
MIR210 & 1 \\
LOC143666 & 0 \\
PHRF1 & 0 \\
IRF7 & 1 \\
CDHR5 & 0 \\
SCT & 0 \\
DRD4 & 0 \\
\hline
\end{tabular}

PPI, protein-protein interaction; RNH1, ribonuclease/angiogenin inhibitor 1; HRAS, HRas proto-oncogene, GTPase; LRRC56, leucine rich repeat containing 56; LMNTD2, lamin tail domain containing 2; RASSF7, Ras association domain family member 7; MIR210, microRNA 210; PHRF1, PHD and ring finger domains 1; IRF7, interferon regulatory factor 7; CDHR5, cadherin related family member 5; SCT, secretin; DRD4, dopamine receptor D4.

were connected to hub genes within the MIR210HG regulatory network, reinforcing our prediction that MIR210HG serves as a transcriptional modulator in colon cancer. Although there is a lack of evidence indicating the specific molecular association between the selected transcription factors and candidates, it was revealed that some transcription factors (including MED15 and Angiopoietin-related protein 4, BCL3 and GAPDH, Cyclin-dependent kinase 9 and HRAS) interact with the hub genes and are strongly correlated with MIR210HG. A total of 5 selected transcription factors were associated with cancer metastasis. Decreased MED15 expression can suppress the transforming growth factor- $\beta /$ Smad signaling

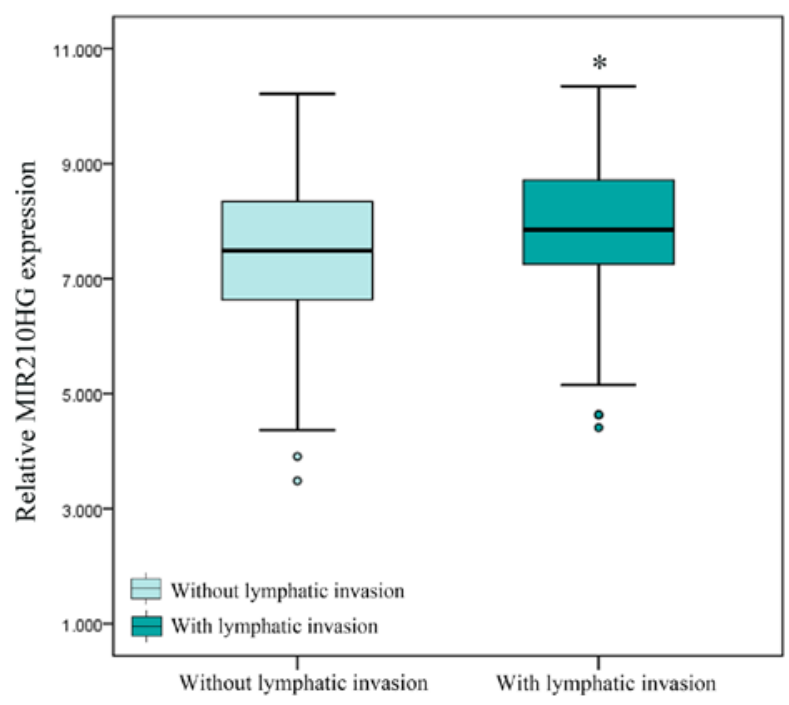

Figure 6. Relative expression level of MIR210HG in patients with or without lymphatic invasion. ${ }^{*} \mathrm{P}<0.05$ vs. without lymphatic invasion.

pathway, thereby downregulating the metastasis of highly invasive breast cancer cells (38). BCL3 can also reduce cancer cell metastasis by influencing cell motility (39). Furthermore, neighboring mRNAs located within $1 \mathrm{Mbps}$ of the MIR210HG loci were investigated to predict possible cis element target genes of MIR210HG. A total of 13 genes were identified, 5 of which, including HRAS, were members of the MIR210HG regulatory network. These results provide new information as how MIR210HG regulates the regulatory network and affects survival in patients with colon cancer.

In summary; in the present study survival-related lncRNAs in colon cancer were screened and bioinformatics analyses were performed to investigate the prognostic potential of MIR210HG and its related genes in colon cancer. The findings indicated that MIR210HG may exert its effects in colon cancer by modulating energy metabolism and cell adhesion. Further predictions suggested that MIR210HG may also affect colon cancer via transcription and post-transcriptional processing. Together, these results provide evidence of the transcriptional regulatory network of MIR $210 \mathrm{HG}$ in colon cancer, and 
suggest that MIR210HG may serve a potential role as a novel biomarker and therapeutic target. Despite the multiple bioinformatic and statistical methods used in the present study, it does have some limitations, and further studies are required to verify these results. Experimental evidence will be provided in future studies; for example, the high expression level of MR210HG may regulate the expression levels of screened MIR210HG-related genes, or induce the phenotype obtained with bioinformatics analyses (such as the metastasis of colon cancer cells), which will be assessed using cell-based assays with colon cancer cell lines.

\section{Acknowledgements}

Not applicable.

\section{Funding}

The present study was supported by grants from the National Natural Science Foundation of China (grant nos. 81760094 and 31602111), the Science and Technology Research Project for Youth of Educational Commission in Jiangxi Province (grant no. GJJ160238), the Science and Technology Foundation for Youths of Jiangxi province (grant no. 20171BAB215021) and the Science and Technology Project of Health and Family Planning commission of Jiangxi Province (grant. no. 20175081).

\section{Availability of data and materials}

The datasets used and/or analyzed during the current study are available from the corresponding author on reasonable request.

\section{Authors' contributions}

YL and ZR designed the study. ZR, ZX and ZL performed the data analyses. All authors contributed to the conception of the study and drafted the manuscript. All authors contributed significantly in writing the manuscript and read and approved the final manuscript.

\section{Ethics approval and consent to participate}

Not applicable.

\section{Patient consent for publication}

Not applicable.

\section{Competing interests}

The authors declare that they have no competing interests.

\section{References}

1. Centers for Disease Control and Prevention (CDC): Vital Signs: Colorectal cancer screening, incidence and mortality-United States, 2002-2010. MMWR Morb Mortal Wkly Rep 60: 884-889, 2011.

2. Okugawa Y, Grady WM and Goel A: Epigenetic alterations in colorectal cancer: Emerging biomarkers. Gastroenterology 149: 1204-1225, 2015.
3. Balaguer F, Link A, Lozano JJ, Cuatrecasas M, Nagasaka T, Boland CR and Goel A: Epigenetic silencing of miR-137 is an early event in colorectal carcinogenesis. Cancer Res 70: 6609-6618, 2010

4. Ponting CP, Oliver PL and Reik W: Evolution and functions of long noncoding RNAs. Cell 136: 629-641, 2009.

5. Zhang LM, Wang P, Liu XM and Zhang YJ: LncRNA SUMO1P3 drives colon cancer growth, metastasis and angiogenesis. Am J Transl Res 9: 5461-5472, 2017.

6. Dianatpour A and Ghafouri-Fard S: The role of long non coding RNAs in the repair of DNA double strand breaks. Int J Mol Cell Med 6: 1-12, 2017.

7. Espinoza CA, Goodrich JA and Kugel JF: Characterization of the structure, function and mechanism of B2 RNA, an ncRNA repressor of RNA polymerase II transcription. RNA 13: 583-596, 2007.

8. Engreitz JM, Haines JE, Perez EM, Munson G, Chen J, Kane M, McDonel PE, Guttman M and Lander ES: Local regulation of gene expression by lncRNA promoters, transcription and splicing. Nature 539: 452-455, 2016.

9. Reinius B, Shi C, Hengshuo L, Sandhu KS, Radomska KJ, Rosen GD, Lu L, Kullander K, Williams RW and Jazin E: Female-biased expression of long non-coding RNAs in domains that escape X-inactivation in mouse. BMC Genomics 11: 614, 2010.

10. Cancer Genome Atlas Research Network: Weinstein JN, Collisson EA, Mills GB, Shaw KR, Ozenberger BA, Ellrott K, Shmulevich I, Sander C and Stuart JM: The cancer genome atlas pan-cancer analysis project. Nat Genet 45: 1113-1120, 2013.

11. Dennis G Jr, Sherman BT, Hosack DA, Yang J, Gao W, Lane HC and Lempicki RA: DAVID: Database for annotation, visualization and integrated discovery. Genome Biol 4: P3, 2003.

12. Huang DW, Sherman BT and Lempicki RA: Bioinformatics enrichment tools: Paths toward the comprehensive functional analysis of large gene lists. Nucleic Acids Res 37: 1-13, 2009.

13. Huang da W, Sherman BT and Lempicki RA: Systematic and integrative analysis of large gene lists using DAVID bioinformatics resources. Nat Protoc 4: 44-57, 2009.

14. Franceschini A, Szklarczyk D, Frankild S, Kuhn M, Simonovic M, Roth A, Lin J, Minguez P, Bork P, von Mering C and Jensen LJ: STRING v9.1: Protein-protein interaction networks, with increased coverage and integration. Nucleic Acids Res 41: D808-D815, 2013.

15. Smoot ME, Ono K, Ruscheinski J, Wang PL and Ideker T: Cytoscape 2.8: New features for data integration and network visualization. Bioinformatics 27: 431-432, 2011.

16. Chin $\mathrm{CH}$, Chen SH, Wu HH, Ho CW, Ko MT and Lin CY: cytoHubba: Identifying hub objects and sub-networks from complex interactome. BMC Syst Biol 8 (Suppl 4): S11, 2014.

17. Nepusz T, Yu H and Paccanaro A: Detecting overlapping protein complexes in protein-protein interaction networks. Nature Methods 9: 471-472, 2012.

18. Li JH, Liu S, Zhou H, Qu LH and Yang JH: starBase v2.0: Decoding miRNA-ceRNA, miRNA-ncRNA and protein-RNA interaction networks from large-scale CLIP-Seq data. Nucleic Acids Res 42: D92-D97, 2014.

19. Muppirala UK, Honavar VG and Drena D: Predicting RNA-protein interactions using only sequence information. BMC Bioinformatics 12: 489, 2011.

20. Li Y, Li Y, Chen W, He F, Tan Z, Zheng J, Wang W, Zhao Q and Li J: NEAT expression is associated with tumor recurrence and unfavorable prognosis in colorectal cancer. Oncotarget 6: 27641-27650, 2015 .

21. Meng J, Li P, Zhang Q, Yang Z and Fu S: A four-long non-coding RNA signature in predicting breast cancer survival. J Exp Clin Cancer Res 33: 84, 2014.

22. Shen Z, Li Q, Deng H, Lu D, Song H and Guo J: Long non-coding RNA profiling in laryngeal squamous cell carcinoma and its clinical significance: Potential biomarkers for LSCC. PLoS One 9: e108237, 2014.

23. Min W, Dai D, Wang J, Zhang D, Zhang Y, Han G, Zhang L, Chen C, Li X, Li Y and Yue Z: Long noncoding RNA miR210HG as a potential biomarker for the diagnosis of glioma. PLoS One 11: e0160451, 2016

24. Xia W, Ren X, Li B, Yue J and Long L: Applying modularity analysis of PPI networks to sequenced organisms. Virulence 3: 459-463, 2012

25. Li J, Wu QM, Wang XQ and Zhang CQ: Long noncoding RNA miR210HG sponges miR-503 to facilitate osteosarcoma cell invasion and metastasis. DNA Cell Biol 36: 1117-1125, 2017. 
26. Serrano M, Lin AW, Mccurrach ME, Beach D and Lowe SW: Oncogenic ras provokes premature cell senescence associated with accumulation of p53 and p16INK4a. Cell 88: 593-602, 1997.

27. Harris GJ, Church JM, Senagore AJ, Lavery IC, Hull TL, Strong SA and Fazio VW: Factors affecting local recurrence of colonic adenocarcinoma. Dis Colon Rectum 45: 1029-1034, 2002.

28. Baxter NN, Virnig DJ, Rothenberger DA, Morris AM, Jessurun J and Virnig BA: Lymph node evaluation in colorectal cancer patients: A population-based study. J Natl Cancer Inst 97: 219-225, 2005.

29. Lin CW, Li XR, Zhang Y, Hu G, Guo YH, Zhou JY, Du J, Lv L, Gao K, Zhang Y and Deng H: TAp63 suppress metastasis via miR-133b in colon cancer cells. Br J Cancer 110: 2310-2320, 2014.

30. Tan X, He X, Jiang Z, Wang X, Ma L, Liu L, Wang X, Fan Z and Su D: Derlin-1 is overexpressed in human colon cancer and promotes cancer cell proliferation. Mol Cell Biochem 408: 205-213, 2015.

31. Yue B, Qiu S, Zhao S, Liu C, Zhang D, Yu F, Peng Z and Yan D: LncRNA-ATB mediated E-cadherin repression promotes the progression of colon cancer and predicts poor prognosis. J Gastroenterol Hepatol 31: 595-603, 2016.

32. Tracy KM, Tye CE, Ghule PN, Malaby HLH, Stumpff J, Stein JL, Stein GS and Lian JB: Mitotically-associated lncRNA (MANCR) affects genomic stability and cell division in aggressive breast cancer. Mol Cancer Res 16: 587-598, 2018.

33. Warburg O: On the origin of cancer cells. Science 123: 309-314, 1956.
34. Song K, Li M, Xu X, Xuan LI, Huang G and Liu Q: Resistance to chemotherapy is associated with altered glucose metabolism in acute myeloid leukemia. Oncol Lett 12: 334-342, 2016.

35. Kawai K, Uemura M, Munakata K, Takahashi H, Haraguchi N, Nishimura J, Hata T, Matsuda C, Ikenaga M, Murata K, et al: Fructose-bisphosphate aldolase A is a key regulator of hypoxic adaptation in colorectal cancer cells and involved in treatment resistance and poor prognosis. Int J Oncol 50: 525-534, 2016.

36. Yang P, Li Z, Fu R, Wu H and Li Z: Pyruvate kinase M2 facilitates colon cancer cell migration via the modulation of STAT3 signalling. Cell Signal 26: 1853-1862, 2014.

37. Zarnack K, Konig J, Tajnik M, Martincorena I, Eustermann S, Stévant I, Reyes A, Anders S, Luscombe NM and Ule J: Direct competition between hnRNP C and U2AF65 protects the transcriptome from the exonization of Alu elements. Cell 152: 453-466, 2013.

38. Zhao M, Yang X, Fu Y, Wang H, Ning Y, Yan J, Chen YG and Wang G: Mediator MED15 modulates transforming growth factor beta (TGF $\beta) / S m a d$ signaling and breast cancer cell metastasis. J Mol Cell Biol 5: 57-60, 2013.

39. Wakefield A, Soukupova J, Montagne A, Ranger J, French R, Muller WJ and Clarkson RW: Bcl3 selectively promotes metastasis of ERBB2-driven mammary tumors. Cancer Res 73: 745-755, 2013.

This work is licensed under a Creative Commons Attribution-NonCommercial-NoDerivatives 4.0 International (CC BY-NC-ND 4.0) License. 